ORIGINAL RESEARCH

\title{
Comorbidities of myasthenic crisis patients according to age and gender in Dr. Soetomo General Academic Hospital, Surabaya, Indonesia in 2017-2019
}

\author{
Andi Muh Rahul Alfaidin ${ }^{1}$, Viskasari P. Kalanjati ${ }^{*} \mathbb{E}$, Mudjiani Basuki $^{{ }^{*} \mathbb{D}}$ \\ ${ }^{1}$ Faculty of Medicine, Universitas Airlangga, Surabaya, Indonesia. \\ ${ }^{2}$ Department of Anatomy, Histology and Pharmacology, Faculty of Medicine, Universitas Airlangga, Surabaya, \\ Indonesia. \\ ${ }^{3}$ Department of Neurology, Faculty of Medicine, Universitas Airlangga; Dr. Soetomo General Academic \\ Hospital, Surabaya, Indonesia.
}

\begin{tabular}{ll}
\hline Article Info & ABSTRACT \\
\hline Article history: & Background: There are limited studies on the comorbidities of \\
Received Jul 29,2021 & myasthenic crisis (MC) associated with age and gender, \\
Revised Nov 17, 2021 & specifically from Indonesia. These data might be valuable for \\
Accepted Des 9, 2021 & treatment to improve the outcome of these patients. Objective: To \\
Published Jan 5, 2022 & analyze the correlation between age and gender with the \\
& comorbidities amongst the MC patients admitted in the ICU of Dr. \\
\hline & Soetomo General Academic Hospital, Surabaya, Indonesia in 2017- \\
Keywords: & 2019. Materials and methods: A retrospective cross-sectional \\
Comorbidities & analytic study was conducted on 25 medical records of MC patients \\
Human \& disease & who fulfilled the inclusion and exclusion study criteria. Descriptive \\
Intensive Care Unit & statistics and Chi-square tests were done to determine the potential \\
Myasthenic Crisis & correlation between variables (significant level of p<0.05). \\
& Results: The age of MC patients was $\leq 50$ years (80\%) and >50 \\
*Corresponding authors & years (20\%) while the gender predominantly found were females \\
Viskasari P. Kalanjati & (68\%) with 32\% males. A significant correlation was found \\
viskasari-p-k@ fk.unair.ac.id & between age and comorbidities (p=0.002) whilst not between \\
Mudjiani Basuki & gender and comorbidities (p=0.115). The most common \\
anibasuki@yahoo.com & comorbidities observed were hypertension (30\%) and diabetes \\
& mellitus (20\%). Conclusion: There were 40\% of patients found \\
& with comorbidities. There was a significant association between \\
& age and comorbidities found amongst myasthenic crisis patients, \\
& whilst no significant association was found between gender and \\
& comorbidities.
\end{tabular}

\footnotetext{
How to cite:

Alfaidin, A.M.R., Kalanjati, V.P., Basuki, M. 2022. Comorbidities of myasthenic crisis patients according to age and gender in Dr. Soetomo General Academic Hospital, Surabaya, Indonesia in 2017-2019. Majalah Biomorfologi, 32(1): 1-5.

Majalah Biomorfologi (Biomorphology Journal) p.ISSN:0215-8833, e.ISSN: 2716-0920 doi: $10.20473 /$ mbiom.v32i1.2022.1-5

cC (i) Copyright: (c) 2022 by the authors. Open access publication under the terms and condition of Copyright: ( ) 2022 by the authors. Open access publication under the terns
the Creative Commons Attribution 4.0 International license (CC.BY 4.0).
} 


\section{BACKGROUND}

Myasthenic crisis (MC) is a complication of myasthenia gravis (MG) that is characterized by worsening muscle weakness especially in respiratory muscles that resulted in need for intubation and mechanical ventilation (Wendell \& Levine, 2011). Myasthenic crisis is found in 15-30\% of patients with MG and usually happened in the early years after diagnosis (Roper, et al., 2017). Myasthenia gravis itself is an autoimmune disease caused by acetylcholine receptor antibodies against a neuromuscular junction of the muscles (Hehir \& Silvestri, 2018). In the United States, the prevalence was predicted to reach 200 per one million population (Berrih-Aknin, et all., 2014). In Indonesia, the epidemiological data on MG or MC has yet to be reported.

Although rare, MG can be found in various ethnicities, geographic locations, and at all ages and genders. Several studies have shown that women are more exposed to MG at the age of fewer than 50 years, whereas between the age of 50 and 60 there is no specific gender predominance. However, after 60 years, MG is more common in males (Berrih-Aknin, et all., 2014). The median interval between $\mathrm{MC}$ and the first onset of MG ranged from 8-12 months. MC may also be the first clinical manifestation in one-fifth of MG patients. The distribution of the incidence of MC shows that before the age of 55 years, women are found to be more exposed, whereas after the age of 55 years the ratio of MC in males and females is approximately similar (Wendell \& Levine, 2011).

Previous studies have shown that MG patients can be found with autoimmune as well as nonautoimmune comorbidities. There are limited studies on the role of comorbidities in MG patients, but these comorbidities may affect the treatment outcome (Sieb, 2014; Misra, et al., 2019). In this study, we reported the comorbidities found in MC patients and analyzed their correlation with the age and the gender of the patients.

\section{OBJECTIVE}

The objective of this study was to study the comorbidities found in myasthenic crisis patients and to analyze their correlation with the age and gender of the MC patients admitted to the Intensive Care Unit of Dr. Soetomo General Academic Hospital, Surabaya, Indonesia in 2017-2019.

\section{MATERIALS AND METHODS}

This study was an analytic observational study with a cross-sectional retrospective method. All the patients included in this study were myasthenic crisis patients admitted to the Intensive Care Unit of Dr. Soetomo General Academic Hospital, Surabaya, Indonesia between January 2017 to December 2019. Data were taken from the medical records of the patients. Incomplete data of medical records was an exclusion criterion. Data obtained was age when admitted to the ICU, gender, and the presence of comorbidities $(\mathrm{n}=25)$. Data were analyzed using a statistical descriptive and Chi-square test with significance value $p<0.05$ (performed by using IBM SPSS Statistic 25.0).

\section{RESULTS}

From this study, we found a total of 25 myasthenic crisis patients admitted to the ICU of Dr. Soetomo General Academic Hospital, Surabaya, Indonesia during 2017-2019. There were 20 patients $(80 \%)$ aged $\leq 50$ years whilst 5 patients $(20 \%)$ aged 50 years or more. In the patients aged $\leq 50$ years, it was found that there were more female patients than male patients with a ratio of 3:1, whereas, in the patients aged 50 years or more, there were more males than females (Table 1).

Table 1. Age and gender of the patients

\begin{tabular}{cccc}
\hline & & \multicolumn{2}{c}{ Age } \\
\cline { 3 - 4 } & & $\leq 50$ years & $>50$ years \\
\hline \multirow{2}{*}{ Gender } & Male $(\mathrm{n}=8)$ & $5(62.5 \%)$ & $3(37.5 \%)$ \\
& Female $(\mathrm{n}=17)$ & $15(88.2 \%)$ & $2(11.8 \%)$ \\
\hline Total $(\mathrm{n}=25)$ & & $20(80 \%)$ & $5(20 \%)$ \\
\hline
\end{tabular}


We found $68 \%$ of the MC patients were females $(n=17)$ and the rests were males $(n=8)$. Out of 25 patients in this study, there were 10 patients with comorbidity. The comorbidities found from this study were hypertension $(n=3)$, diabetes mellitus $(n=2)$, thymoma $(n=1)$, aspiration pneumonia $(n=1)$, atelectasis $(n=1)$, ARDS $(n=1)$, low hemoglobin level $(n=1)$, and liver abscess $(n=1)$. There was one patient with more than one comorbidity (Table 2).

Table 2. Comorbidities found amongst all patients

\begin{tabular}{lc}
\hline Comorbidities & Total \\
\hline Hypertension & 2 \\
Diabetes mellitus + hypertension & 1 \\
Diabetes mellitus & 1 \\
Thymoma & 1 \\
Aspiration pneumonia & 1 \\
Atelectasis & 1 \\
ARDS & 1 \\
Low haemoglobin level & 1 \\
Liver abscess & 1 \\
\hline
\end{tabular}

We found a significant association between comorbidities and age in the MC patients $(p=0.002)$. However, we observed that there was no significant association between comorbidities and gender amongst these patients $(p=0.115)$ (Table 3$)$.

Table 3. Correlation between comorbidities with age and gender

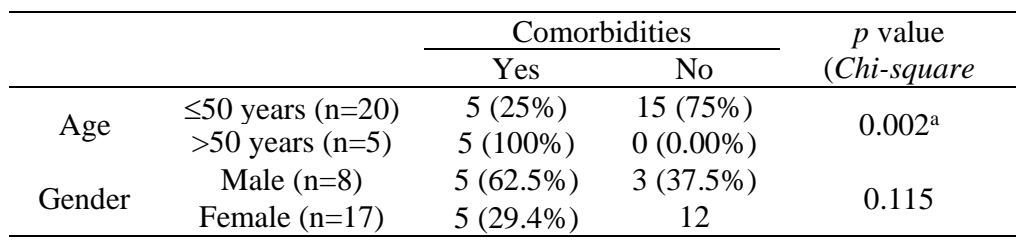

${ }^{\mathrm{a}}$ Significant

\section{DISCUSSION}

In this study, we found that the myasthenic crisis patients were predominantly aged $\leq 50$ years (80\%) and most were females (68\%). From the study of Liu et al., (2016) conducted amongst 33 patients in China, it was reported that the myasthenic crisis more commonly occurred in females. This result was consistent with our study in Indonesia where the socio-demography status was quite similar compared to that in China. Another study by Lv et al., (2019), which was conducted amongst 78 patients, also reported similar results to the prior study. However, studies by Kalita et al., (2014) in India and Neumann et al., (2019) in Germany reported different results where the predominant gender found was male. The age and gender of myasthenic crisis patients were represented in a bimodal distribution. In patients aged $\leq 55$ years, it was found that there were more females than a male with a ratio of $4: 1$, while at the age 50 years or more the ratio was equal (Wendell \& Levine, 2011). This was found to be quite similar with the results of our study wherein amongst patients aged $\leq 50$ there were more female than male. But in patients aged 50 years or more, the results of our study were quite different from the prior study (Chen, et al., 2020). Patients of myasthenia gravis with comorbidities nowadays tend to seek medical health earlier, thus comorbidities would be relatively controlled. This could affect the prevalence of myasthenic crisis.

In our study, we found that there was a significant correlation between age and comorbidities amongst these patients, although there was no significant correlation between gender and these comorbidities. Alexander, et al., (2019) reported that age was significantly correlated to the comorbidities amongst the myasthenic crisis patients. The comorbidities reported in this study included cardiac illness such as myocardial stunning, cardiac arrhythmias and pulmonary diseases such as bronchopneumonia. This study was conducted amongst myasthenic crisis patients in the Neuro-Intensive Care Unit (ICU) of a tertiary care center in South India, which was likely similar with the settings of our study. From the study of Misra, et al., (2019), it was reported that $88 \%$ of the 
patients had miscellaneous comorbidities such as diabetes, hypertension, and metabolic syndrome. These results were especially common in patients with late-onset MG (aged $>50$ years), similar with the results of our study. Another study by Wong et al., (2016) also reported similarity to the result where the most comorbidities found were hypertension followed by diabetes mellitus and hyperlipidemia. Epidemiological studies have confirmed that the incidence of MG increases in both older men and women is due to increased life expectancy. This may lead to increased comorbidities related to the old age of these patients (Farrugia \& Goodfellow, 2020). This is of course a challenge for both patients and clinicians because it will complicate whether the patients' health problems are caused by myasthenia gravis itself or comorbidities not related to the myasthenia gravis (Gilhus, et al., 2014).

Myasthenic crisis is a life-threatening medical condition that requires early diagnosis. Management of MC is primarily done by providing respiratory assistance in the form of intubation and mechanical ventilation. However, the mortality rate in $\mathrm{MC}$ has improved significantly due to developments in respiratory care management and intensive care units (Lal, et al., 2013). This situation has led to a longer life expectancy of patients with MG, which allows exploring and discovering the comorbidities in these patients. Studies conducted on comorbidities of patients with MG resulted in a tendency towards autoimmune disorder, but a different type of comorbidities has also been found (Misra, et al., 2019). Comorbidity in patients with MG has been said to be an important aspect seeing that the conditions are highly linked to the MG subgroup, which classifies MG based on the distinctive autoantibodies and clinical symptoms (Gilhus, et al., 2014; Nacu, et al., 2015). Comorbidities, accompanied by other factors such as ICU complications, therapy options, and treatment side effects may be crucial for the quality of life of patients with MG (Gilhus, et al., 2014). These comorbid diseases may also influence the outcome of the MG patients who suffered from the crisis, thus comorbidities in these patients should be examined and treated thoroughly.

\section{CONCLUSION}

This study showed that the myasthenic crisis patients were predominantly aged $\leq 50$ years $(80 \%)$ and $68 \%$ were females. There were $40 \%$ of patients with comorbidities, with the commonest comorbidities being hypertension and diabetes mellitus. There was a strong correlation between age and comorbidities found amongst these patients although not the case between gender and comorbidities.

\section{REFERENCES}

Alexander, M., Sivadasan, A., Aaron, S., Mathew, V., Nair, S., Muthusamy, K., Prabhakar, A., Benjamin, R., Shaikh, A., Rynjah, G., 2019. Comorbidities and long-term outcomes in a cohort with myasthenic crisis: Experiences from a tertiary care center. Annals of Indian Academy of Neurology, 22(4): 464-471. doi: 10.4103/aian.AIAN_197_19.

Berrih-Aknin, S., Frenkian-Cuvelier, M. and Eymard, B., 2014. Diagnostic and clinical classification of autoimmune myasthenia gravis. Journal of Autoimmunity, 48-49: 143-148. doi: 10.1016/j.jaut.2014.01.003.

Chen, J., Tian, D., Zhang, C., Li, Z., Zhai, Y., Xiu, Y., Gu, H., Li, H., Wang, Y., Shi, F., 2020. Incidence, mortality, and economic burden of myasthenia gravis in China: A nationwide populationbased study. The Lancet Regional Health - Western Pacific, 5. doi: 10.1016/j.lanwpc.2020.100063.

Farrugia, M. and Goodfellow, J., 2020. A practical approach to managing patients with myasthenia gravis-opinions and a review of the literature. Frontiers in Neurology, 11. doi: 10.3389.fneur.2020.00604.

Gilhus, N., Nacu, A., Andersen, J., Owe, J., 2014. Myasthenia gravis and risks for comorbidity. European Journal of Neurology, 22(1): 17-23. doi: 10.1111/ene/12599.

Hehir, M. and Silvestri, N., 2018. Generalized Myasthenia Gravis. Neurologic Clinics, 36(2): 253260. doi: 10.1016/j.ncl.2018.01.002.

Kalita, J., Kohat, A., Misra, U., 2014. Predictors of outcome of myasthenic crisis. Neurological sciences: official journal of the Italian Neurological Society and of the Italian Society of Clinical Neurophysiology, 35(7): 11-14. doi: 10.1007/s10072-014-1659-y. 
Lal, V., Prabhakar, S., Agarwal, R., Sharma, S., 2013. Clinical profile and outcome of myasthenic crisis in a tertiary care hospital: a prospective study. Annals of Indian Academy of Neurology, 16(2): 203.doi: 10.4103/0972-2327.112466.

Liu, Z., Yao, S., Zhou, Q., Deng, Z., Zou, J., Feng, H., Zhu, H., Cheng, C., 2016. Predictors of extubation outcomes following myasthenic crisis. Journal of International Medical Research, 44(6): 1524-1533. doi: 10.1177/0300060516669893.

Lv, Z., Zhong, H., Huan, X., Song, J., Yan, C., Zhou, L., Lu, J., Zhu, W., Lin, J., Qiao, K., Xu, Y., Lu, J., Xi, J., Luo, S., Zhao, C., 2019. Predictive score for in-hospital mortality of myasthenic crisis: a retrospective Chinese cohort study. European Neurology, 81(5-6): 287-293. doi: 10.1159/000503961.

Misra, U., Kalita, J., Singh, V., Kumar, S., 2019. A study of comorbidities in myasthenia gravis. Acta Neurologica Belgica, 120(1): 59-64. doi: 10.1007/s13760-019-011-2-w.

Nacu, A., Andersen, J., Lisnic, V., Owe, J., Gilhus, N., 2015. Complicating autoimmune diseases in myasthenia gravis: a review. Autoimmunity, 48(6): 362-368. doi: 10.3109/08916934.2015.1030614.

Neumann, B., Angstwurm, K., Mergenthaler, P., Kohler, S., Schönenberger, S., Bösel, J., Neumann, U., Vidal, A., Huttner, H., Gerner, S., Thieme, A., Steinbrecher, A., Dunkel, J., Roth, C., Schneider, H., Schimmel, E., Fuhrer, H., Fahrendorf, C., Alberty, A., Zinke, J., Meisel, A., Dohmen, C., Stetefeld, H., 2019. Myasthenic crisis demanding mechanical ventilation. Neurology, 94(3): e299e313. doi: 10.1212/WNL.0000000000008688.

Roper, J., Fleming, M., Long, B., Koyfman, A., 2017. Myasthenia gravis and crisis: evaluation and management in the emergency department. The Journal of Emergency Medicine, 53(6): 843-853. doi: 10.1016/j/jemermed.2017.06.009.

Sieb, J., 2014. Myasthenia gravis: an update for the clinician. Clinical \& Experimental Immunology, 175(3): 408-418. doi: 10.1111/cei.12217.

Wendell, L. and Levine, J., 2011. Myasthenic crisis. The Neurohospitalist, 1(1): 16-22. doi: $10.1177 / 1941875210382918$.

Wong, Y., Ong, C., Sung, S., Wu, C., Hsu, Y., Su, Y. \& Hung, L., 2016. Clinical profile and outcome of myasthenic crisis in Central Taiwan. Acta Neurol Taiwan, 25(4): 129-135. 